\title{
Thermal and energetic processing of astrophysical ice analogues rich in $\mathrm{SO}_{2}$
}

\author{
Z. Kaňuchová ${ }^{1,3}$, Ph. Boduch ${ }^{2}$, A. Domaracka ${ }^{2}$, M. E. Palumbo ${ }^{3}$, H. Rothard ${ }^{2}$, and G. Strazzulla ${ }^{3}$ \\ 1 Astronomical Institute of Slovak Academy of Sciences, 05960 Tatranská Lomnica, Slovakia \\ e-mail: pipovci@gmail.com \\ 2 Centre de Recherche sur les Ions, les Matériaux et la Photonique, Normandie Univ, ENSICAEN, UNICAEN, CEA, CNRS, CIMAP, \\ 14000 Caen, France \\ 3 INAF-Osservatorio Astrofisico di Catania, via Santa Sofia 78, 95123 Catania, Italy
}

Received 28 February 2017 / Accepted 8 June 2017

\begin{abstract}
Context. Sulfur is an abundant element in the cosmos and it is thus an important contributor to astrochemistry in the interstellar medium and in the solar system. Astronomical observations of the gas and of the solid phases in the dense interstellar/circumstellar regions have evidenced that sulfur is underabundant. The hypothesis to explain such a circumstance is that it is incorporated in some species in the solid phase (i.e. as frozen gases and/or refractory solids) and/or in the gas phase, which for different reasons have not been observed so far.

Aims. Here we wish to give a contribution to the field by studying the chemistry induced by thermal and energetic processing of frozen mixtures of sulfur dioxide (one of the most abundant sulfur-bearing molecules observed so far) and water.

Methods. We present the results of a series of laboratory experiments concerning thermal processing of different $\mathrm{H}_{2} \mathrm{O}: \mathrm{SO}_{2}$ mixtures and ion bombardment $\left.(30 \mathrm{keV} \mathrm{He})^{+}\right)$of the same mixtures. We used in situ Fourier transform infrared (FTIR) spectroscopy to investigate the induced effects.

Results. The results indicate that ionic species such as $\mathrm{HSO}_{3}^{-}, \mathrm{HSO}_{4}^{-}$, and $\mathrm{S}_{2} \mathrm{O}_{5}^{2-}$ are easily produced. Energetic processing also produces $\mathrm{SO}_{3}$ polymers and a sulfurous refractory residue.

Conclusions. The produced ionic species exhibit spectral features in a region that, in astronomical spectra of dense molecular clouds, is dominated by strong silicate absorption. However, such a dominant feature is associated with some spectral features, some of which have not yet been identified. We suggest adding the sulfur-bearing ionic species to the list of candidates to help explain some of those features. In addition, we suggest that once expelled in the gas phase by sublimation, due to the temperature increase, and/or by non-thermal erosion those species would constitute a class of molecular ions not detected so far. We also suggest that molecular sulfur-bearing ions could be present on the surfaces and/or in the atmospheres of several objects in the solar system, for example icy satellites of the giant planets and comets.
\end{abstract}

Key words. astrochemistry - methods: laboratory: molecular - techniques: spectroscopic - ISM: molecules - dust, extinction

\section{Introduction}

Atomic sulfur is an abundant element in the cosmos. Its abundance relative to hydrogen is about $1.32 \times 10^{-5}$ (Asplund et al. 2009) and is thus an important contributor to chemical evolution in the galaxies and in the solar system. However, many astronomical observations (both of the gas and of the solid phases) in the dense interstellar medium (ISM) and in star forming regions have evidenced that sulfur is underabundant, i.e. the sum of sulfur atoms locked in the sulfur-bearing molecules detected so far only accounts for a fraction of its cosmic abundance.

As an example, Tieftrunk et al. (1994) summed up the abundances of $\mathrm{SO}, \mathrm{CS}, \mathrm{SO}_{2}$, and $\mathrm{H}_{2} \mathrm{~S}$, which are the most abundant $\mathrm{S}$-bearing molecules observed in the gas phase both in lowand high-density molecular clouds. They concluded that these molecules only account for a fraction of the sulfur abundance in the cosmos, of the order of $10^{-3}$. In the solid phase, only OCS (Palumbo et al. 1995, 1997; Ferrante et al. 2008) and $\mathrm{SO}_{2}$ (Boogert et al. 1997) have been detected so far in icy grain mantles toward high-mass protostars. Their estimated abundances are low, however, and can account for only about $0.5 \%$, and 0.8 $4.0 \%$, respectively, of the total sulfur abundance (Boogert et al. 1997; Palumbo et al. 1997).
Thus, the problem of the missing sulfur is a hot question in astrochemistry. It is obvious to postulate that sulfur is incorporated in some species - either in the solid phase and/or in the gas phase - which for different reasons have not yet been observed. It is important to note that this lack concerns the dense interstellar medium only. In diffuse clouds the amount of gas phase sulfur fully accounts for the total sulfur abundance and rules out the possibility of its depletion on refractory interstellar grains (see e.g. Sofia et al. 1994).

In this context some studies have been performed to try to understand which species, although not yet observed in the solid phase, could be present, and then, once expelled to the gas phase by thermal or non-thermal processes, could be searched for in the gas phase. It is believed that the desorption of grain mantle species into the gas phase, for example after warming by a protostar or sputtering by energetic cosmic ions, gives an important contribution to the gas phase composition (Modica \& Palumbo 2010; Palumbo et al. 2008). As outlined by Martín-Doménech et al. (2016) it seems plausible that an important fraction of the observed S-bearing gas phase species is released from grains because molecules such as $\mathrm{H}_{2} \mathrm{~S}, \mathrm{SO}_{2}$, OCS, SO, $\mathrm{H}_{2} \mathrm{CS}, \mathrm{HCS}^{+}$, and $\mathrm{NS}$ have abundances that cannot 
be explained with gas-phase-only chemical models (Doty et al 2004; Viti et al. 2004; Wakelam et al. 2011; Woods et al. 2015).

A molecule that has been considered in particular detail is $\mathrm{H}_{2} \mathrm{~S}$. This is a very important point because as soon as the medium recondenses (toward the formation of molecular clouds), the high abundance of hydrogen easily produces hydrogenated solid species such as $\mathrm{H}_{2} \mathrm{O}, \mathrm{CH}_{4}$, and $\mathrm{NH}_{3}$ which are, indeed, well observed. It is then puzzling that this is not the case for $\mathrm{H}_{2} \mathrm{~S}$ (Garrod et al. 2007; Vidal et al. 2017). At present it is not clear whether this is due to observational difficulties. The next generation of instruments, namely the James Webb Space Telescope (JWST) could clarify the question. In the meantime, a number of laboratory experiments simulating the energetic processing of icy mantles on grains in the ISM have demonstrated that energetic processing of solid $\mathrm{H}_{2} \mathrm{~S}$ by ions and photons (UV, $\mathrm{X}$-rays) produce sulfur-sulfur bonds $\left(\mathrm{H}_{2} \mathrm{~S}_{2}\right.$ and $\mathrm{HS}_{2}$ are easily formed) and also a refractory polymer-like residue (Grim \& Greenberg 1987; Moore et al. 2007; Garozzo et al. 2010; Jiménez-Escobar et al. 2012, 2014).

It has been suggested that part of the missing sulfur could be in solid unvolatile refractory grains (Garozzo et al. 2010; Jiménez-Escobar \& Muñoz Caro 2011) or it could be released in the gas phase as $\mathrm{H}_{2} \mathrm{~S}_{2}, \mathrm{HS}_{2}, \mathrm{~S}_{2}$ (Jiménez-Escobar \& Muñoz Caro 2011), or $\mathrm{CS}_{2}$ (formed when mixtures of $\mathrm{H}_{2} \mathrm{~S}$ and $\mathrm{CO}$ are irradiated in the laboratory, Garozzo et al. 2010). This would also imply that hydrogen sulfide is not detected since it is easily transformed into different species.

These findings stimulated recent efforts to observe $\mathrm{H}_{2} \mathrm{~S}_{2}$, $\mathrm{HS}_{2}$, and $\mathrm{S}_{2}$ in the gas phase toward the low-mass warm core IRAS 16293-2422 (Martín-Doménech et al. 2016). Estimated upper limit abundances of these molecules are up to two orders of magnitude lower than the $\mathrm{H}_{2} \mathrm{~S}$ abundance in the source. This possibly indicates that gas-phase chemistry after their desorption from the icy mantles efficiently destroys those species.

With the aim of contributing to the field, we present here the results of a series of experiments conducted at the laboratories of the Centre de recherche sur les Ions, les MAtériaux et la Photonique (CIMAP)-Grand Accélérateur National d'Ions Lourds (GANIL) in Caen (France) and at the Laboratorio di Astrofisica Sperimentale (LASp) in Catania (Italy). The experiments conducted at CIMAP-GANIL concern the thermal processing of different mixtures $\mathrm{H}_{2} \mathrm{O}: \mathrm{SO}_{2}$ and the implantation of multicharged sulfur ions in water ice; the experiments conducted in Catania are relative to ion bombardment $\left(30 \mathrm{keV} \mathrm{He}^{+}\right)$of the same mixed species. The results indicate that ionic species such as $\mathrm{HSO}_{3}^{-}, \mathrm{HSO}_{4}^{-}$, and $\mathrm{S}_{2} \mathrm{O}_{5}^{2-}$ are produced by the three processes (thermal, S-implantation in pure water ice, and ion bombardment) and we suggest that they have to be searched for in the inter- and circumstellar regions where they could contribute to the inventory of the missing sulfur atoms.

Our experiments are also relevant to some objects in the solar system, namely Jupiter's Galilean satellites (see e.g. Dalton et al. 2010). In particular, frozen $\mathrm{SO}_{2}$ is the dominant species at the surface of Io, and it was also observed in cometary comae (see e.g. Crovisier \& Bockelée-Morvan 1999). Our results are therefore discussed also in the light of their relevance for these objects.

\section{Experimental procedure}

The experiments conducted at CIMAP-GANIL concern the thermal processing of different mixtures of $\mathrm{H}_{2} \mathrm{O}: \mathrm{SO}_{2}$. The frozen samples were prepared by condensing opportune mixtures of water and sulfur dioxide gases on a CsI window at $16 \mathrm{~K}$. A fine valve allowed the deposition rate to be controlled. A nozzle was used to transmit the gas into the high vacuum chamber and onto the cold CsI substrate installed in the centre of the chamber on a cold finger connected to a closed-cycle helium cryostat. The pressure in the high vacuum chamber was below $10^{-7}$ mbar. The temperature of the substrate was controlled by a carbon resistance and a compound linear thermal sensor (CLTS) situated on the holder, providing a precision of $0.1 \mathrm{~K}$. After deposition the samples were heated up at a rate of about $1 \mathrm{~K} / \mathrm{min}$ and IR spectra taken at the chosen temperatures in the spectral range 5000 $600 \mathrm{~cm}^{-1}(2-16.7 \mu \mathrm{m})$ with a resolution of $1 \mathrm{~cm}^{-1}$. To this end, a Nicolet Magna 550 Fourier Transform Infrared Spectrometer (FTIR) was used. The spectra are taken in transmittance, at normal incidence, and were corrected by a background spectrum recorded before deposition (for more details on the experimental set up, see Ding et al. 2013).

In the experiments conducted in Catania, $\mathrm{H}_{2} \mathrm{O}: \mathrm{SO}_{2}(1: 2)$ mixtures were accreted onto a cold $(16 \mathrm{~K})$ silicon substrate in a vacuum chamber $\left(P<10^{-7}\right.$ mbar $)$. Infrared transmittance spectra (resolution of $1 \mathrm{~cm}^{-1}$ ) were obtained, before and after $30 \mathrm{keV}$ $\mathrm{He}^{+}$ion bombardment, by a Bruker Equinox 55 FTIR spectrometer. Ion beams were produced by an ion implanter (Danfysik 1080-200) and irradiated the sample on a spot greater than the area probed by the infrared beam (for more details on the experimental set up, see Strazzulla et al. 2001; Allodi et al. 2013). As usual in this kind of experiment the molecular ratio of the irradiated mixture is different from that expected in space, which is often dominated by water ice. This is due to the experimental need for a sufficient number of mother molecules to produce the daughter species.

It is important to note that the stoichiometry of a deposited mixture can be evaluated only approximately. In all of the studied mixtures (i.e. at CIMAP-GANIL and in Catania) we evaluated the column density of the deposited species $\left(\mathrm{H}_{2} \mathrm{O}\right.$ and $\left.\mathrm{SO}_{2}\right)$ from infrared spectroscopy. The results significantly differ from the nominal gas mixtures that we prepared before accretion onto the cold finger. This is due to the different thermodynamic properties of the deposited species.

The IR bands of a given molecule were used to measure the column density $N$ in units of molecules $\mathrm{cm}^{-2}$ through the formula

$N=\frac{\int \tau(v) \mathrm{d} v}{A}$,

where $\tau(v)$ is the optical depth (which is 2.3 times the absorbance plotted in the figures) at wavenumber $v\left(\mathrm{~cm}^{-1}\right)$ and $A$ is the band strength $\left(\mathrm{cm}\right.$ molecule $\left.{ }^{-1}\right)$.

The used band strength values are given in Table 1 together with band peak positions and assignment. The band strength values are valid for pure species, and using them to evaluate the column density of each molecule in a mixture introduces a large error that can be as high as $50 \%$.

\section{Results}

\subsection{Energetic processing}

As an example of the results obtained after ion irradiation of frozen mixtures of water or sulfur dioxide, in Fig. 1 we show the spectra of a deposited $(16 \mathrm{~K}) \mathrm{H}_{2} \mathrm{O}: \mathrm{SO}_{2}(1: 2)$ ice mixture that had a thickness $\sim 0.53 \mu \mathrm{m}$, roughly half of the penetration depth of the incoming $30 \mathrm{keV} \mathrm{He}{ }^{+}$ions calculated by the Stopping and Range of Ions in Matter (SRIM) software (Ziegler et al. 2008). Also shown is the spectrum obtained after irradiation with 
Table 1. Peak positions, vibration modes, molecule assignments, and strength of the bands used to calculate the column density of the deposited $\mathrm{H}_{2} \mathrm{O}$ and $\mathrm{SO}_{2}$ mixtures.

\begin{tabular}{lccccl}
\hline \hline $\begin{array}{l}\text { Peak position } \\
\mathrm{cm}^{-1}\end{array}$ & Vibration & Assignment & $\begin{array}{c}\text { Band strength } \\
\times 10^{-17} \mathrm{~cm} \mathrm{~mol}^{-1}\end{array}$ & Ref. \\
\hline 1149 & 8.703 & $v_{1}$ & $\mathrm{SO}_{2}$ & 0.22 & Garozzo et al. (2008) \\
1335 & 7.491 & $v_{3}$ & $\mathrm{SO}_{2}$ & 1.47 & Garozzo et al. (2008) \\
1660 & 6.024 & $v_{2}$ & $\mathrm{H}_{2} \mathrm{O}$ & 1.2 & Gerakines et al. (1995) \\
3280 & 3.045 & $v_{1}, v_{3}$ & $\mathrm{H}_{2} \mathrm{O}$ & 14 & Hagen et al. (1981) \\
\hline
\end{tabular}

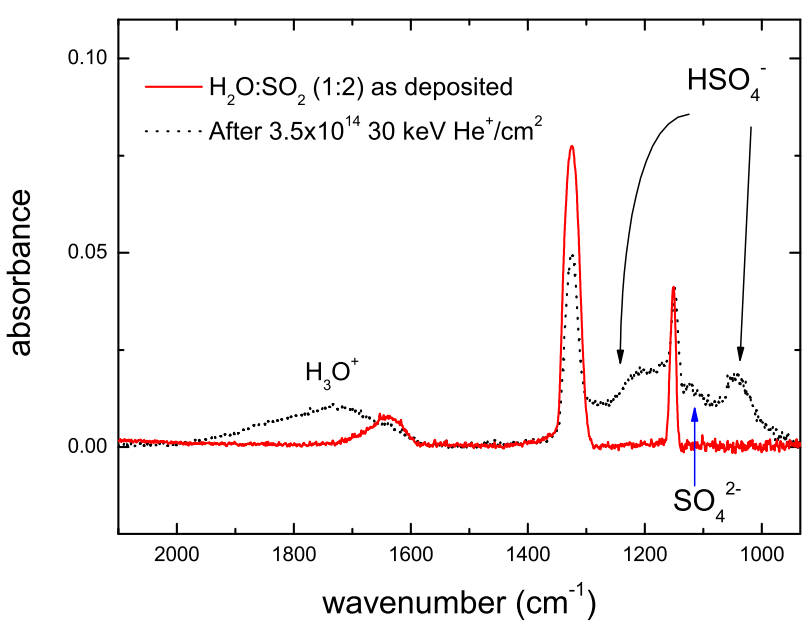

Fig. 1. Infrared transmittance spectra of $\mathrm{H}_{2} \mathrm{O}: \mathrm{SO}_{2}=1: 2$ ice mixture as deposited at $T=16 \mathrm{~K}$ and after $30 \mathrm{keV} \mathrm{He}{ }^{+}$ion irradiation (the given fluence corresponds to a deposited dose of $12.3 \mathrm{eV} / 16 \mathrm{u}$ ). We note that the broad band around $1200 \mathrm{~cm}^{-1}$ is due to more than one species, including $\mathrm{SO}_{3}$ sulfur polymers (see Table 2).

$3.5 \times 10^{14} 30 \mathrm{keV} \mathrm{He} / \mathrm{cm}^{2}$. By using the stopping power of the incoming ions as calculated by the SRIM software (Ziegler et al. 2008) we find that the fluence corresponds to a deposited energy (dose) of $12.3 \mathrm{eV} / 16 \mathrm{u}$ (for details see Strazzulla et al. 2001).

From Fig. 1 it can be easily seen that the intensity of the two $\mathrm{SO}_{2}$ bands centred at $1325 \mathrm{~cm}^{-1}$ and $1150 \mathrm{~cm}^{-1}$ diminishes after irradiation. More precisely, the column density of sulfur dioxide decreases from about $5 \times 10^{17}$ molecules $\mathrm{cm}^{-2}$ to about $3 \times 10^{17}$ molecules $\mathrm{cm}^{-2}$. A fraction of $\mathrm{SO}_{2}$ molecules (and of $\mathrm{H}_{2} \mathrm{O}$ as well) was used to build up new species. In fact, several new bands appeared in the spectrum after ion irradiation (see also Fig. 2). We can observe the formation of the $\mathrm{SO}_{3}$ polymeric chains testified by the presence of the broad band centred at $1200 \mathrm{~cm}^{-1}$ (Moore et al. 2007). Features of sulfate $\mathrm{SO}_{4}^{2-}$ and bisulfate $\mathrm{HSO}_{4}^{-}$ions are observed, as are features of the counter-ion $\mathrm{H}_{3} \mathrm{O}^{+}$. The results are consistent with the previous findings of Moore et al. (2007) who observed the same bands after $800 \mathrm{keV}$ proton irradiation of $\mathrm{H}_{2} \mathrm{O}: \mathrm{SO}_{2}=3: 1$ and 30:1 mixtures (at $T=86 \mathrm{~K}, T=110 \mathrm{~K}$, and $T=132 \mathrm{~K}$ ). The peak position of the bands of newly formed sulfur-bearing species observed in the present experiments and in those available in the literature (Moore et al. 2007) are listed in Table 2.

The experiments described so far are relative to ions whose penetration depth is greater than the thickness of the irradiated layers, as usually occurs with ice mantles on dust grains in interand circumstellar environments irradiated by cosmic ions. There are, however, many instances in which the thickness of the irradiated icy layers is much greater than the penetration depth of the ions that remain implanted in the target. This is the case of most of the icy objects in the solar system (e.g. satellites of the giant planets, comets, Pluto). Implanted ions - if they are reactive, like carbon and sulfur ions - have the chance to form molecular species that include the projectile (see e.g. Strazzulla 2011; Ding et al. 2013; Lv et al. 2013).

Relevant to this paper are the results obtained by Ding et al. (2013) concerning the implantation of $\mathrm{S}^{q+}(q=7,9,11)$ ions at an energy range between 35 and $176 \mathrm{keV}$ in water ice at $80 \mathrm{~K}$ and aimed at simulating the complexity of the irradiation environment to which the surface of icy satellites of the giant planets, particularly Europa, are exposed being embedded in the planetary magnetospheres. The experiments, performed at the lowenergy ion beam facility ARIBE of GANIL in Caen (France), indicate that implantation produces hydrated sulfuric acid with yields that increase with ion energy. The identification was due to the appearance, in the IR spectra of implanted targets, of a broad feature characterized by three maxima around $1135 \mathrm{~cm}^{-1}$, $1105 \mathrm{~cm}^{-1}$, and $1070 \mathrm{~cm}^{-1}$ (see the spectrum in the upper panel of Fig. 2). Following Loeffler et al. (2011) the three observed peaks were assigned to $\mathrm{H}_{2} \mathrm{SO}_{4}$, to $\mathrm{HSO}_{4}^{-}$in monohydrate, and to $\mathrm{SO}_{4}^{2-}$ in tetrahydrate.

\subsection{Thermal processing}

Three samples of icy mixtures with the $\mathrm{H}_{2} \mathrm{O}: \mathrm{SO}_{2}$ concentration ratios 1:10, 1:1, and 3:1 were deposited at $16 \mathrm{~K}$ and then warmed step by step up to $T=160 \mathrm{~K}$. A blank experiment of pure $\mathrm{SO}_{2}$ was also performed. Infrared spectra were taken at low temperature and at various steps during the heating of the samples. Spectra taken at $120 \mathrm{~K}$ are plotted in the bottom panel of Fig. 2. Moore et al. (2007), guided by the works of Zhang \& Ewing (2002) and Fink \& Sill (1982), suggest that the peaks at 1035 and $1011 \mathrm{~cm}^{-1}$ are probably due to the bisulfite ion $\mathrm{HSO}_{3}^{-}$ and either one of its reaction products or an isomer. Another absorbance peak present at around $956 \mathrm{~cm}^{-1}$ (see Fig. 2) is attributed to $\mathrm{S}_{2} \mathrm{O}_{5}^{2-}$, meta-bisulfite (Pichler et al. 1997; Moore et al. 2007). Positions of observed absorption peaks were measured (see Fig. 3) and compared with the position of absorption bands identified in the similar heating experiments of $\mathrm{H}_{2} \mathrm{O}: \mathrm{SO}_{2}$ icy mixtures performed by Moore et al. (2007) and later by Loeffler et al. (2010) on different ratios of the same mixture. Our finding for 1:1 and 3:1 mixtures are in excellent agreement with the results of Moore et al. (2007). However, in the experiment of Moore et al. (2007) with the mixture 30:1 (i.e. with the lowest $\mathrm{SO}_{2}$ concentration), the absorption feature of the bisulfite ion is located at about $1070-1060 \mathrm{~cm}^{-1}$. We do not observe a peak in this region at low $\mathrm{T}$ for a mixture 1:10 (i.e. with the highest $\mathrm{SO}_{2}$ concentration), but only in the spectra taken at high temperature ( $120 \mathrm{~K}$ and above). This could be explained as being due to the sublimation of $\mathrm{SO}_{2}$ and to the drop in its initial high concentration (see Fig. 4). 


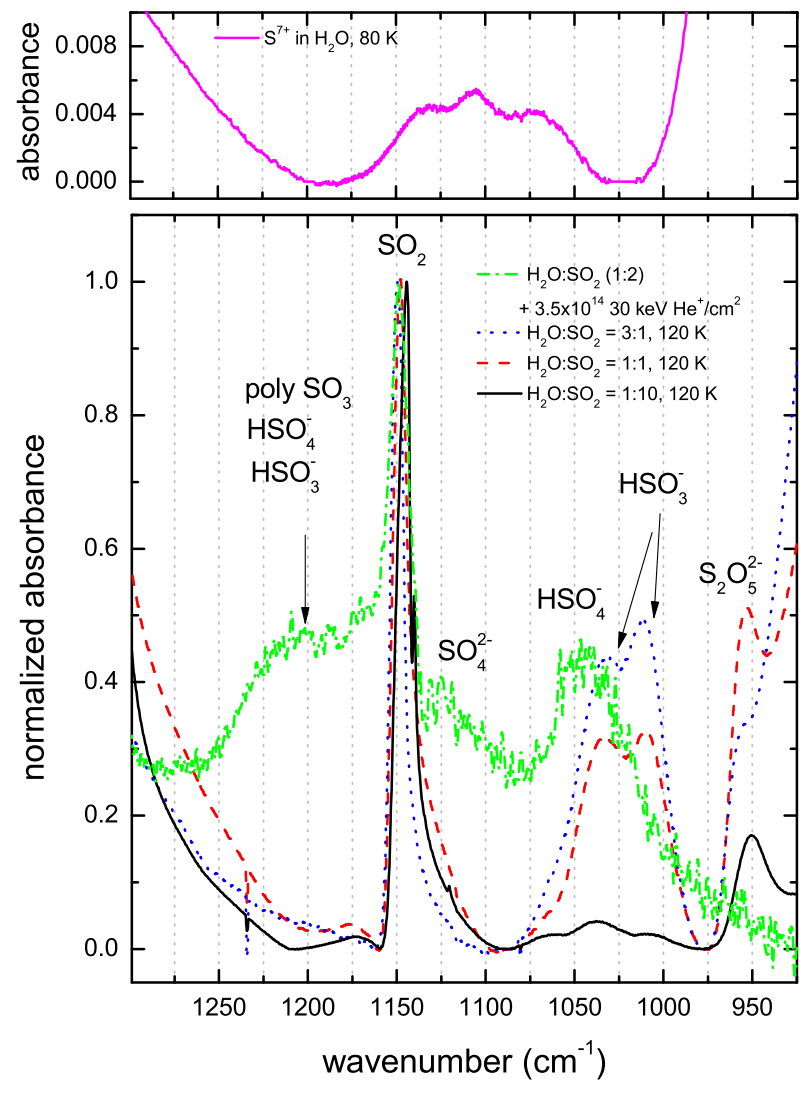

Fig. 2. Comparison of the results obtained by three different kinds of ice processing: thermal processing of different $\mathrm{H}_{2} \mathrm{O}: \mathrm{SO}_{2}$ mixtures, ion bombardment $\left(30 \mathrm{keV} \mathrm{He}^{+}\right)$of the same mixed species, and implantation of $\mathrm{S}^{7+}$ ions in water ice.

Because we do not know the band strengths of the newly formed bands, we are not able to measure the column density of the species. However, assuming that the band strength values do not depend on the temperature, we can deduce the fate of sulfur dioxide and sulfite ions in the mixtures by measuring the band areas with increasing temperature. The band areas of all relevant features and the relative area of the $\mathrm{SO}_{2}$ band at $1149 \mathrm{~cm}^{-1}$ were measured and they are plotted in Fig. 4 . For the newly formed bands, we in fact measured a small initial value for the band areas (in agreement with the finding by Moore et al. 2007 and Loeffler et al. 2010) that we attribute to the thermal reactions induced by the water latent heat of condensation of water ice. When pure $\mathrm{SO}_{2}$ ice is heated, its sublimation occurs at $120 \mathrm{~K}$ as evidenced by a drop in the relative absorption band area (Fig. 4). When $\mathrm{SO}_{2}$ ice is mixed with water ice, the area of the $1149 \mathrm{~cm}^{-1}$ band decreases well before $120 \mathrm{~K}$. Together with the decrease in the $\mathrm{SO}_{2}$ band area, the sulfite absorption feature (1035-1065 $\mathrm{cm}^{-1}$ ) grows with the temperature up to $120 \mathrm{~K}$, after which it is lost as the samples are further warmed. Thus, before it sublimates, about $50 \%$ of the sulfur dioxide was used by thermal reactions with $\mathrm{H}_{2} \mathrm{O}$ for the formation of sulfur-bearing ionic species. At temperatures $\geq 120 \mathrm{~K}$, the sublimation of $\mathrm{SO}_{2}$ takes place and the newly formed species follow the same fate. The rate (efficiency) of the formation of ionic species slightly depends on the relative concentrations of the two ices in the mixture as shown in the two bottom panels of Fig. 4. We also notice that because of a higher number of water molecules surrounding the $\mathrm{SO}_{2}$ molecules, some of these latter are trapped and sublimate at higher temperatures, as is commonly observed for many other icy mixtures (e.g. Collings et al. 2004).

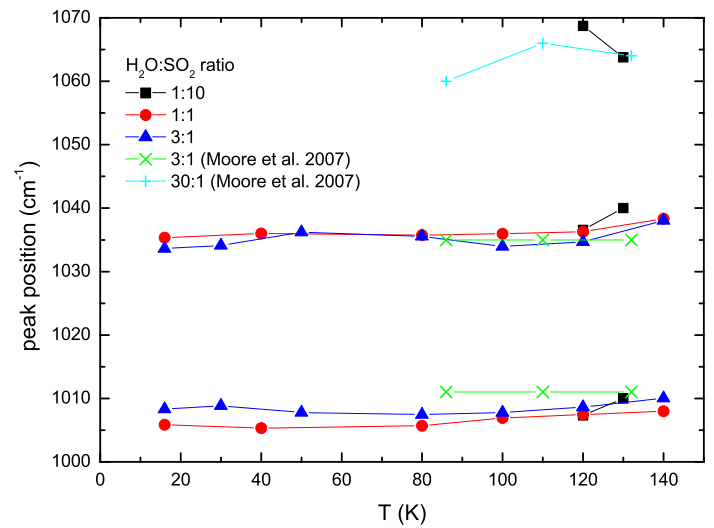

Fig. 3. Peak positions of bisulfite ions $\left(\mathrm{HSO}_{3}^{-}\right)$in different $\mathrm{H}_{2} \mathrm{O}: \mathrm{SO}_{2}$ ice mixtures during thermal processing.
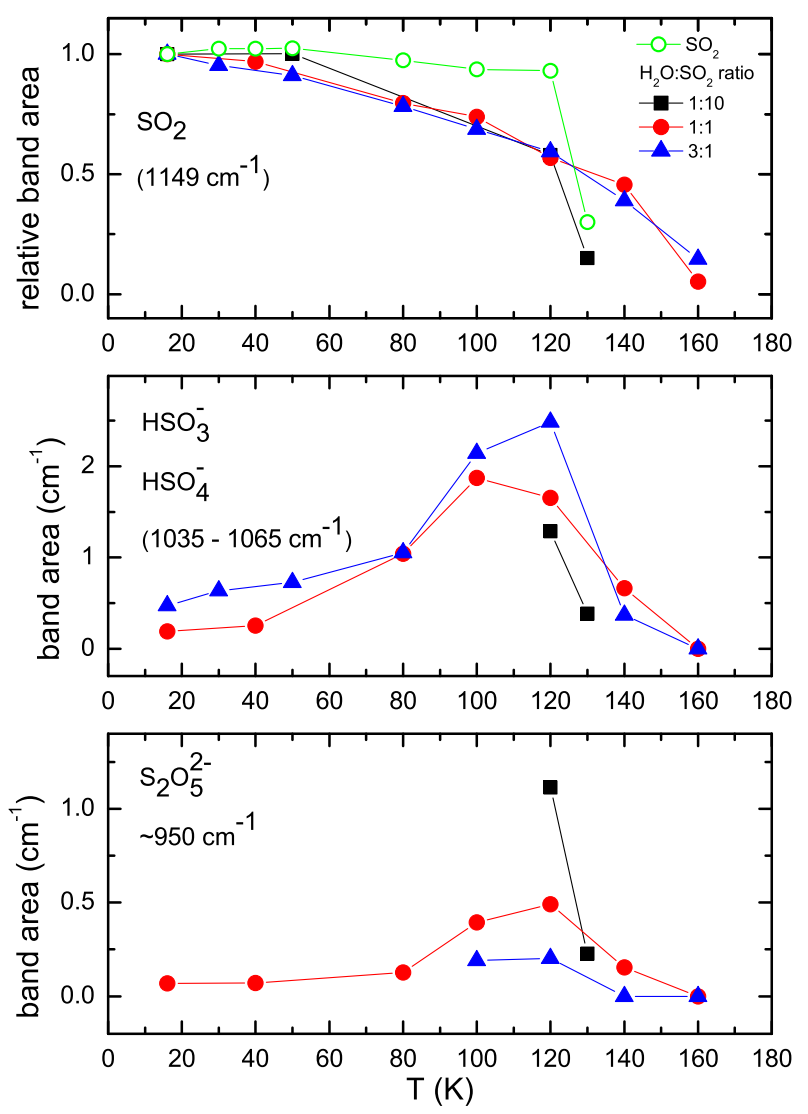

Fig. 4. Variations of the relative $\mathrm{SO}_{2}$ band area (top panel) and band areas of sulfite and sulfate ions in different $\mathrm{H}_{2} \mathrm{O}: \mathrm{SO}_{2}$ ice mixtures during thermal processing.

\subsection{Energetic vs. thermal processing}

The spectra of $\mathrm{H}_{2} \mathrm{O}: \mathrm{SO}_{2}$ mixtures processed thermally and by ion bombardment, and the spectrum of pure water ice after $\mathrm{S}^{7+}$ implantation are plotted together in Fig. 2 for comparison. The overview of the bands associated with these molecules for all analysed experiments is given in Table 2 . We note that sulfate and bisulfate ions are the result of radiolytic processes, while bisulfite and meta-bisulfite are produced by thermal processing. This finding can be reciprocally confirmed and explained by the comparison with the results of non-radiolytic, thermally driven 
Table 2. Overview of the position and assignment of the peaks identified in the spectra of various processed mixtures.

\begin{tabular}{llll}
\hline \hline $\begin{array}{l}\text { Peak position } \\
\mathrm{cm}^{-1}\end{array}$ & Assignment & Process/Ice & Ref. \\
\hline 611 & $\mathrm{SO}_{4}^{2-}$ & $\mathrm{H}^{+} \rightarrow \mathrm{H}_{2} \mathrm{O}: \mathrm{SO}_{2}(3: 1 ; 30: 1)$ & $\begin{array}{l}\text { Moore et al. (2007) } \\
\text { this work }\end{array}$ \\
891 & $? ? ?$ & $\mathrm{He}^{+} \rightarrow \mathrm{H}_{2} \mathrm{O}: \mathrm{SO}_{2}(1: 2)$ & this work \\
950 & $\mathrm{~S}_{2} \mathrm{O}_{5}^{2-}$ & thermal, $\mathrm{H}_{2} \mathrm{O}: \mathrm{SO}_{2}(1: 1)$ & Moore et al. (2007) \\
956 & $\mathrm{~S}_{2} \mathrm{O}_{5}^{2-}$ & thermal, $\mathrm{H}_{2} \mathrm{O}: \mathrm{SO}_{2}(3: 1 ; 30: 1)$ & Moore et al. (2007) \\
982 & $\mathrm{SO}_{4}^{2-}$ & $\mathrm{H}^{+} \rightarrow \mathrm{H}_{2} \mathrm{O}: \mathrm{SO}_{2}(3: 1)$ & Moore et al. (2007); this work \\
$1005-1011$ & $\mathrm{HSO}_{3}^{-}$ & thermal, $\mathrm{H}_{2} \mathrm{O}: \mathrm{SO}_{2}(3: 1 ; 1: 10)$ & Moore et al. (2007); this work \\
$1035-1037$ & $\mathrm{HSO}_{3}^{-}$ & thermal, $\mathrm{H}_{2} \mathrm{O}: \mathrm{SO}_{2}(3: 1 ; 1: 1 ; 1: 10)$ & this work \\
1044 & $\mathrm{HSO}_{3}^{-}, \mathrm{HSO}_{4}^{-}$ & $\mathrm{He}^{+} \rightarrow \mathrm{H}_{2} \mathrm{O}: \mathrm{SO}_{2}(1: 2)$ & Moore et al. (2007) \\
1052 & $\mathrm{HSO}_{4}^{-}$ & $\mathrm{H}^{+} \rightarrow \mathrm{H}_{2} \mathrm{O}: \mathrm{SO}_{2}(3: 1 ; 30: 1)$ & this work \\
$1060-1065$ & $\mathrm{HSO}_{3}^{-}$ & thermal, $\mathrm{H}_{2} \mathrm{O}: \mathrm{SO}_{2}(1: 10)$ & Ding et al. (2013) \\
1070 & $\mathrm{SO}_{4}^{2-}$ & implantation, $\mathrm{S}^{7+} \rightarrow \mathrm{H}_{2} \mathrm{O}$ & Ding et al. (2013) \\
1105 & $\mathrm{HSO}_{4}^{-}$ & implantation, $\mathrm{S}^{7+} \rightarrow \mathrm{H}_{2} \mathrm{O}$ & Moore et al. (2007) \\
1110 & $\mathrm{SO}_{4}^{2-}$ & $\mathrm{H}^{+} \rightarrow \mathrm{H}_{2} \mathrm{O}: \mathrm{SO}_{2}(3: 1 ; 30: 1)$ & this work \\
1120 & $\mathrm{SO}_{4}^{2-} ?$ & $\mathrm{He}^{+} \rightarrow \mathrm{H}_{2} \mathrm{O}: \mathrm{SO}_{2}(1: 2)$ & Ding et al. (2013) \\
1135 & $\mathrm{H}_{2} \mathrm{SO}_{4}$ & implantation, $\mathrm{S}^{7+} \rightarrow \mathrm{H}_{2} \mathrm{O}$ & this work \\
1200 & poly SO$_{3}$ & $\mathrm{He}^{+} \rightarrow \mathrm{H}_{2} \mathrm{O}: \mathrm{SO}_{2}(1: 2)$ & Moore et al. (2007) \\
1235 & $\mathrm{HSO}_{3}^{-}, \mathrm{HSO}_{4}^{-}$ & $\mathrm{H}^{+} \rightarrow \mathrm{H}_{2} \mathrm{O}: \mathrm{SO}_{2}(3: 1 ; 30: 1)$ & \\
\hline
\end{tabular}

experiments (Loeffler et al. 2013, 2016). Loeffler et al. (2013) performed experiments by heating frozen $\mathrm{H}_{2} \mathrm{O}: \mathrm{SO}_{2}: \mathrm{H}_{2} \mathrm{O}_{2}$ mixtures, the last component being the main product of water ice radiolysis. They found that sulfate ions are produced when $\mathrm{H}_{2} \mathrm{O}_{2}$ is present, in contrast to what happens after thermal processing of the binary mixture $\mathrm{H}_{2} \mathrm{O}: \mathrm{SO}_{2}$. Thus, for the binary mixture we have

$\mathrm{SO}_{2}+\mathrm{H}_{2} \mathrm{O} \rightarrow \mathrm{HSO}_{3}^{-}+\mathrm{H}^{+}$.

By adding $\mathrm{H}_{2} \mathrm{O}_{2}$ (product of radiolysis) this is rapidly followed by

$\mathrm{H}_{2} \mathrm{O}_{2}+\mathrm{HSO}_{3}^{-}+\mathrm{H}^{+} \rightarrow \mathrm{HSO}_{4}^{2-}+\mathrm{H}_{3} \mathrm{O}^{+}$.

It is relevant to mention that Loeffler et al. (2013) used these results as a possible explanation for some of the observations related to the presence and distribution of hydrogen peroxide across Europa's surface and of its lack on Ganymede and Callisto.

Similarly Loeffler et al. (2016) studied the thermal processing of the solid mixture $\mathrm{H}_{2} \mathrm{O}: \mathrm{SO}_{2}: \mathrm{O}_{3}$. They demonstrated that thermally driven reactions in solid phase occur below $150 \mathrm{~K}$, and the main sulfur-bearing species is bisulfate. They also suggested that $\mathrm{SO}_{2}$ and $\mathrm{O}_{3}$ on the surface of the icy Jovian satellites will efficiently react making detection of these molecules in the same vicinity unlikely.

\section{Discussion}

\subsection{Protostellar regions}

As already said, the only sulfur-bearing molecules observed in the solid phase toward high-mass protostars are OCS (Palumbo et al. 1995, 1997) and $\mathrm{SO}_{2}$ (Boogert et al. 1997), but they have low abundances and can account only for a minor amount of the elemental sulfur. Nevertheless, the presence of $\mathrm{SO}_{2}$ in the icy mantles in protostellar regions has stimulated the experiments presented here, aimed at investigating which additional sulfurbearing species are formed after energetic and/or thermal processing of sulfur dioxide mixed with water ice. Our finding that ionic species such as $\mathrm{HSO}_{3}^{-}, \mathrm{HSO}_{4}^{-}$, and $\mathrm{S}_{2} \mathrm{O}_{5}^{2-}$ are produced raises two questions: (1) are they observed/observable in the interstellar medium in the solid phase and/or in the gas phase after they are desorbed from the icy mantles because of thermal and/or non-thermal mechanisms? and (2) can they give a significant contribution to the inventory of sulfur species?

In order to find an answer to the question of the presence of the ionic sulfur-bearing species in the solid phase, we have given a look at the literature and made a comparison between our experimental results and the observations by Lacy et al. (1998) relative to the infrared spectra of four embedded protostars in the $750-1230 \mathrm{~cm}^{-1}$ range. This spectral region is dominated by the very intense silicate band that complicates the detection of possible further contributing species. However, Lacy et al. (1998) were able to detect, for NGC 7538 IRS9, a band at $1110 \mathrm{~cm}^{-1}$ that they attributed to frozen ammonia in a polar water-rich interstellar ice, and several others near 785, 820, 900, 1030 , and $1075 \mathrm{~cm}^{-1}$ that were unidentified. Later, the band at $1030 \mathrm{~cm}^{-1}$ was confirmed by ISO observations and attributed to frozen methanol (Gibb et al. 2004). Unfortunately, a fitting procedure between astronomical and laboratory spectra is not feasible in the present case. In fact, the profile of the observed features, which overlap with the dominant silicate band, cannot be well defined. In addition, the relative intensities of the possibly observed features cannot be reproduced by a single laboratory spectrum. Each one should be treated as a single feature, but this would introduce an indetermination that is too strong. Therefore, here we can only outline that the sulfur species which we identify in laboratory spectra are in a spectral region where there are several unidentified bands whose peak positions are coincident or very close to those measured in the laboratory. Future astronomical observations (e.g. by the JWST) could help to clarify their attribution. In particular, it would be interesting to observe MonR2-IRS3 (or similar sources), a source warm enough to have caused the sublimation of the most volatile species and retained the less volatile ones (Schutte \& Khanna 2003).

The lack of an appreciable amount of $\mathrm{SO}_{2}$ in the observed spectra gives some insight into the chemical pathway that drives the formation of sulfur-bearing species on icy mantles. It is in 
fact thought that sulfur atoms accreting on grains are mostly hydrogenated and should produce $\mathrm{H}_{2} \mathrm{~S}$; however, this is not observed. As suggested by Garozzo et al. (2010) this could be due to energetic processing by cosmic ions that in presence of oxygen and carbon bearing species (e.g. $\mathrm{CO}$ ) easily converts hydrogenated sulfur into other species, including $\mathrm{SO}_{2}$. However, the further ion processing of $\mathrm{SO}_{2}$ in the presence of oxygen and carbon bearing species reduces the amount of $\mathrm{SO}_{2}$ in favour of other species such as those investigated here. In addition, the lack of $\mathrm{SO}_{2}$ after sulfur implantation into water ice has already been evidenced by Ding et al. (2013). Those authors outlined that $\mathrm{SO}_{2}$ is formed by the addition of $\mathrm{S}$ to $\mathrm{O}_{2}\left(\mathrm{and} /\right.$ or $\left.\mathrm{HO}_{2}\right)$, but it is easily converted to hydrated sulfuric acid via

$\mathrm{SO}_{2}+\mathrm{H}_{2} \mathrm{O}_{2} \rightarrow \mathrm{H}_{2} \mathrm{SO}_{4}$.

In other words, atomic sulfur that hits a grain surface is efficiently converted by energetic and thermal processes to more complex sulfur-bearing species rather than being accumulated as sulfur dioxide. In this scenario, we can suggest that these species contribute to the inventory of sulfur-bearing species, but at present it is not possible to establish whether their abundance can significantly contribute to solving the question of the missing sulfur because we are not able, due to the lack of suitable band strength values and the paucity of observational evidences, to measure the column density of these species in the solid phase. In addition, these ionic species and the fragments of $\mathrm{SO}_{3}$ polymers have not yet been observed in the gas phase after their sublimation when the temperature increases, for example in the regions nearer to the forming star. We hope that the results presented here stimulate the search for these species in the gas phase in opportune environments by using the ALMA facility, for example. Adequate laboratory studies on the relevant spectral line parameters of the sulfur-bearing species are also necessary.

\subsection{Solar system objects}

The experimental results presented here are relevant to a number of objects in the solar system where sulfur dioxide has been observed or is presumed to be present. These objects include Jupiter's Galilean satellites Io, Europa, Ganymede, and Callisto. Being embedded in the Jovian magnetosphere, they are exposed to the complex flux of low- (plasma) and high-energy electron and ion bombardment (Dalton et al. 2010).

Io's surface is in fact dominated by sulfur dioxide, which is expelled from the very intense volcanic activity triggered by tidal effects (Peale et al. 1979). Although it is thought that Io has lost nearly all of its hydrogen (Zolotov \& Fegley 1999), the detection of hydrogen pickup ions by Galileo's plasma analyser (Frank \& Paterson 1999) in the space surrounding the satellite raised the question regarding its origin. A first suggestion was hydrogen sulfide (Nash \& Howell 1989). However, the abundance of this compound is very low (with an upper limit of $10^{-4}$ ) with respect to $\mathrm{SO}_{2}$ (Schmitt \& Rodriguez 2003). The next candidates as hydrogen bearing species are then water ice and/or hydrate materials whose absorption bands around $3150 \mathrm{~cm}^{-1}$ were tentatively observed (Salama et al. 1984; Carlson et al. 1997). It is, however, also possible that the detected flux of hydrogen ions comes from the Jovian magnetosphere and not from the satellite.

In this scenario, and assuming that hydrogen bearing species are present on Io's surface along with the certain presence of intense fluxes of energetic ions and electrons, radiolytic products are likely to be present. The most abundant should be dehydrated species such as the fragments of the elemental sulfur residue formed after ion bombardment of pure sulfur dioxide
(Gomis \& Strazzulla 2008). The sulfur residue could be responsible of the observed red slope in the near-infrared/visible spectral region of Io's spectra and of the molecular fragments $S_{4}$ and $\mathrm{S}_{8}$ (see e.g. Fig. 4 in Dalton et al. 2010). Much less abundant (and difficult to observe) are the hydrated ions that we have synthesized in the experiments described here; nevertheless, they merit further investigation.

The present experiments are of primary relevance to the remaining three water ice dominated Galilean satellites and for the other icy satellites orbiting Jupiter, Saturn (e.g. Enceladus), and Uranus. As already mentioned above, Ding et al. (2013) have demonstrated experimentaly that magnetospheric sulfur ions implanted in Europa's surface produce hydrated sulfuric acid. The production rate is high enough to explain the quantity of hydrated sulfuric acid on the surface of Europa inferred to be present by modelling the near infrared $(2 \mu \mathrm{m})$ water ice band (Dalton et al. 2013) as observed by Galileo the Near-Infrared Mapping Spectrometer (NIMS). However, the bands due to hydrated sulfuric acid in the laboratory spectra of sulfur-implanted water ice targets appear in the $1100 \mathrm{~cm}^{-1}$ region (see Fig. 2 and Table 2). Such a spectral region has been investigated by instruments on board Voyager and Cassini and will be investigated by instruments on board the James Webb Space Telescope (JWST). The data collected by flyby and orbiter missions is incomplete and/or collected under imperfect illumination conditions (e.g. high phase angles). Therefore, the contribution of JWST will be relevant and we suggest that a particular effort should be made to identify the hydrated sulfuric acid features, particularly on Europa which exhibits surface regions exposed to very intense fluxes of energetic sulfur ions (Dalton et al. 2010). sulfurbearing ions should also be searched for in the exospheres of the icy satellites where they could be expelled by thermal and non-thermal processes.

Our experiments are also relevant to comets and to all of the small objects in the outer solar system (transNeptunian objects). Several sulfur-bearing species have already been observed in different families of comets (see e.g. Crovisier \& Bockelée-Morvan 1999; Crovisier 2006).

The question of the type and abundance of sulfur-bearing species was revised by Calmonte et al. (2016) based on the results obtained by the Rosetta Orbiter Spectrometer for Ion and Neutral Analysis/Double Focusing Mass Spectrometer in the coma of comet 67P/Churyumov-Gerasimenko. Those authors measured the abundances of the species that were previously known to be present on comets, namely $\mathrm{H}_{2} \mathrm{~S}, \mathrm{OCS}, \mathrm{SO}, \mathrm{S}_{2}, \mathrm{SO}_{2}$, and $\mathrm{CS}_{2}$. Calmonte et al. (2016) detected $\mathrm{S}_{3}, \mathrm{~S}_{4}, \mathrm{CH}_{3} \mathrm{SH}$, and $\mathrm{C}_{2} \mathrm{H}_{6} \mathrm{~S}$ for the first time, and they concluded that the derived total elemental sulfur abundance of 67P does not show any sulfur depletion. In addition, those authors presented results indicating that sulfur-bearing species have been processed by radiolysis in the pre-solar cloud and that at least some of the ice from this cloud has survived in comets up to the present. This conclusion is in fact based on experimental results that show how ion irradiation of sulfur-bearing species produce a solid unvolatile sulfurrich residue (Gomis \& Strazzulla 2008; Woods et al. 2015) and also molecules originally not present such as $\mathrm{CS}_{2}$ (formed when mixtures $\mathrm{H}_{2} \mathrm{~S}$ and $\mathrm{CO}$ are irradiated in the laboratory, Garozzo et al. 2010) and the ionic species discussed here that we suggest should be searched for.

\section{Conclusion}

In this paper we have described the results of a series of experiments concerning thermal and energetic processing of $\mathrm{SO}_{2}$ ices 
mixed with water ice. The results indicate that ionic species such as $\mathrm{HSO}_{3}^{-}, \mathrm{HSO}_{4}^{-}$, and $\mathrm{S}_{2} \mathrm{O}_{5}^{2-}$ are formed. Ion bombardment also produces $\mathrm{SO}_{3}$ polymers and a sulfur-rich refractory residue. The results have been discussed in view of their potential relevance to the debate on the missing sulfur in the interstellar and circumstellar regions, and to the chemical evolution of the surfaces of icy objects in the solar system. The results can be summarized as follows:

- We suggest that sulfur-bearing ionic species could be synthesized on interstellar icy grain mantles by energetic or thermal processes. These species and the fragments of $\mathrm{SO}_{3}$ polymers sublimate when the temperature increases, for example in the regions close to the forming star. This finding should stimulate theoretical, observational, and experimental researches. It is in fact important to theoretically investigate the contribution of the ionic species expelled in the gas phase to the chemistry of those regions. At the same time experimental efforts to measure the rotational spectra of these molecules are necessary in order to allow the observers to identify them through astronomical observations.

- Hydrated sulfuric acid formed after sulfur ion implantation in water ice produces alterations of the shape of its $2 \mu \mathrm{m}$ band as already observed (Dalton et al. 2010). It also gives origin to a multi-peaked band in the mid-IR spectral region (Ding et al. 2013), which should be searched for in the spectra of waterdominated solid surfaces. sulfur-bearing ionic species should be searched for in the gas phase after being released from the surface by thermal and/or non-thermal processes.

- On Io it is possible that non-hydrated sulfur-bearing species have already been observed. Only a small amount of hydrated sulfur-bearing species is predicted to be present on Io's surface, if any.

- The inventory of sulfur-bearing molecules recently implemented by the Rosetta finding (Calmonte et al. 2016) supports evidence for the occurrence of radiolysis. If so, that inventory would be even more implemented including the species discussed here.

Considering the ensemble of the results presented here, and in agreement with recent findings by the NASA-Goddard group (e.g. Loeffler et al. 2016) the present work suggests that the combined effect of thermal and radiolytic processes plays a fundamental role in driving the chemical evolution of sulfur-bearing ices.

Acknowledgements. This work was supported by the Italian Ministero dell'Istruzione, dell'Università e della Ricerca through the grant Progetti Premiali 2012-iALMA (CUP C52I13000140001). G.S. was supported by the Italian Space Agency (ASI 2013-056 JUICE Partecipazione Italiana alla fase A/B1) and by the European COST Action CM1401-Our Astrochemical History. Z.K. was supported by VEGA - The Slovak Agency for Science, Grant No. 2/0032/14. This work was also supported by COST Action TD1308 - ORIGINS

\section{References}

Allodi, M. A., Baragiola, R. A., Baratta, G. A., et al. 2013, Space Sci. Rev., 180, 101

Asplund, M., Grevesse, N., Sauval, A. J., \& Scott, P. 2009, ARA\&A, 47, 481

Boogert, A. C. A., Schutte, W. A., Helmich, F. P., Tielens, A. G. G. M., \& Wooden, D. H. 1997, A\&A, 317, 929
Calmonte, U., Altwegg, K., Balsiger, H., et al. 2016, MNRAS 462, S253

Carlson, R. W., Smythe, W. D., Lopes-Gautier, R. M. C., et al. 1997, Geophys. Res. Lett., 24, 2479

Collings, M. P., Anderson, M. A., Chen, et al. 2004, MNRAS 354, 1133

Crovisier J. 2006, Mol. Phys., 104, 2737

Crovisier, J., \& Bockelée-Morvan, D. 1999, Space Sci. Rev., 90, 19

Dalton, J. B., Cruikshank, D. P., Stephan, K., et al. 2010, Space Sci. Rev., 153, 113

Dalton, J. B., Cassidy, T., Paranicas, C., et al. 2013, Planet. Space Sci., 77, 45

Ding, J. J., Boduch, P., Domaracka, A., et al. 2013, Icarus, 226, 860

Doty, S. D., van Dishoeck, E. F., \& Tan, J. 2004, BAAS, 36, 1505

Ferrante, R. F., Moore, M. H., Spiliotis, M. M., \& Hudson, R. L. 2008, ApJ, 684, 1210

Fink, U., \& Sill, G. T. 1982, The infrared spectral properties of frozen volatiles, ed. L. Wilkening (Tucson: Univ. of Arizona Press), 164

Frank, L. A., \& Paterson, W. R. 1999, JRG, 104, 28657

Garrod, R. T., Wakelam, V., \& Herbst, E. 2007, A\&A, 467, 1103

Garozzo, M., Fulvio, D., Gomis, O., Palumbo, M. E., \& Strazzulla, G. 2008, Planet. Space Sci., 56, 1300

Garozzo, M., Fulvio, D., Kaňuchová, Z., Palumbo, M. E., \& Strazzulla, G. 2010, A\&A, 509, A67

Gerakines, P. A., Schutte, W. A., Greenberg, J. M., \& van Dishoeck, E. F. 1995, A\&A, 296, 810

Gibb, E. L., Whittet, D. C. B., Boogert, A. C. A., \& Tielens, A. G. G. M̀. 2004, ApJS, 151, 35

Gomis, O., \& Strazzulla, G. 2008, Icarus 194, 146

Grim, R. J. A., \& Greenberg, J. M. 1987, A\&A, 181, 155

Hagen, W., Tielens, A. G. G. M., \& Greenberg, J. M. 1981, Chem. Phys., 56, 367

Jiménez-Escobar A., \& Muñoz Caro, G. M. 2011, A\&A, 536, A91

Jiménez-Escobar A., Muñoz Caro, G. M., Cicarelli, A., et al. 2012, ApJ, 751, 343

Jiménez-Escobar A., Muñoz Caro, G. M., \& Chen, Y.-J. 2014, MNRAS, 443, 343

Lacy, J. H., Faraji, H., Sandford, S. A., \& Allamandola, L. J. 1998, ApJ, 501, L105

Loeffler, M. J., \& Hudson, R. L. 2010, Geophys. Res. Lett., 37, L19201

Loeffler, M. J., \& Hudson, R. L. 2013, Icarus, 224, 257

Loeffler, M. J., \& Hudson, R. L. 2016, ApJ, 833, L9

Loeffler, M. J., Hudson, R. L., Moore, M. H., \& Carlson, R. W. 2011, Icarus, 215,270

Lv, X. Y., Boduch, P., Ding, J. J., et al. 2014, MNRAS, 438, 922

Martín-Doménech, R., Jiménez-Serra, I., Munoz Caro, G. M., et al. 2016, A\&A, 585, A112

Modica, P., \& Palumbo, M. E. 2010, A\&A, 519, A22

Moore, M. H., Hudson, R. L., \& Carlson, R. W. 2007, Icarus, 189, 409

Nash, D. B., \& Howell, R. R. 1989, Science, 244, 454

Palumbo, M. E., Tielens, A. G. G. M., \& Tokunaga, A. T. 1995, ApJ, 449, 674

Palumbo, M. E., Geballe, T. R., \& Tielens, A. G. G. M. 1997, ApJ, 479, 839

Palumbo, M. E., Leto, P., Siringo, C., \& Trigilio, C. 2008, ApJ, 685, 1033

Peale, S. J., Cassen, P., \& Reynolds, R. T. 1979, Science, 203, 892

Pichler, A., Fleissner, G., Hallbrucker, A., Mayer, E., 1997, J. Mol. Struct. 408, 521

Salama, F., Allamandola, L. J., Sandford, S. A., et al. 1994, Icarus, 107, 413

Schmitt, B., \& Rodriguez, J. 2003, JGR, 108, 5104

Schutte, W. A., \& Khanna, R. K. 2003, A\&A, 98, 1049

Sofia, U. J., Cardelli, J. A., \& Savage, B. D. 1994, ApJ, 430, 650

Strazzulla, G. 2011, NIMB, 269, 842

Strazzulla, G., Baratta, G. A., Palumbo, M. E. 2001, Spectrochim. Acta A, 57, 825

Tieftrunk, A., Pineau des Forets, G., Schilke, P., \& Walmsley, C. M. 1994, A\&A, 289,579

Vidal, T. H. G., Loison, J.-C., Jaziri, Y. A., et al. 2017, MNRAS, 469, 435

Viti, S., Collings, M. P., Dever, J. W., McCoustra, M. R. S., \& Williams, D. A. 2004, MNRAS, 354, 1141

Wakelam, V., Hersant, F., \& Herpin, F. 2011, A\&A, 529, A112

Woods, P. M., Occhiogrosso, A., Viti, S., et al. 2015, MNRAS, 450, 1256

Zhang, Z., \& Ewing, G. E. 2002, Spectrochim. Acta, 58, 2105

Ziegler J. F., Biersack, J. P., \& Ziegler M. D. 2008, The stopping and range of ions in solids (New York: Pergamon Press)

Zolotov, M. J., \& Fegley, Jr., B. 1999, Icarus, 141, 40 